\title{
Super-chiral vibrational spectroscopy with metasurfaces for high-sensitive identification of alanine enantiomers
}

Cite as: Appl. Phys. Lett. 117, 101103 (2020); https://doi.org/10.1063/5.0012331

Submitted: 30 April 2020 . Accepted: 31 August 2020. Published Online: 09 September 2020

Takumi lida, Atsushi Ishikawa (D), Takuo Tanaka (D), Atsuya Muranaka (D), Masanobu Uchiyama (D), Yasuhiko Hayashi (D), and Kenji Tsuruta (D)
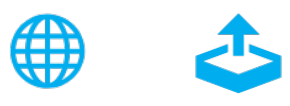

\section{ARTICLES YOU MAY BE INTERESTED IN}

Narrow-band and tunable intense terahertz pulses for mode-selective coherent phonon excitation

Applied Physics Letters 117, 101101 (2020); https://doi.org/10.1063/5.0015612

Sensitive detection of excited energy levels in rare-earth optical materials by a magnetooptical resonant excitation technique

Applied Physics Letters 117, 101102 (2020); https://doi.org/10.1063/5.0016068

Spin current generation and detection in uniaxial antiferromagnetic insulators

Applied Physics Letters 117, 100501 (2020); https://doi.org/10.1063/5.0022391

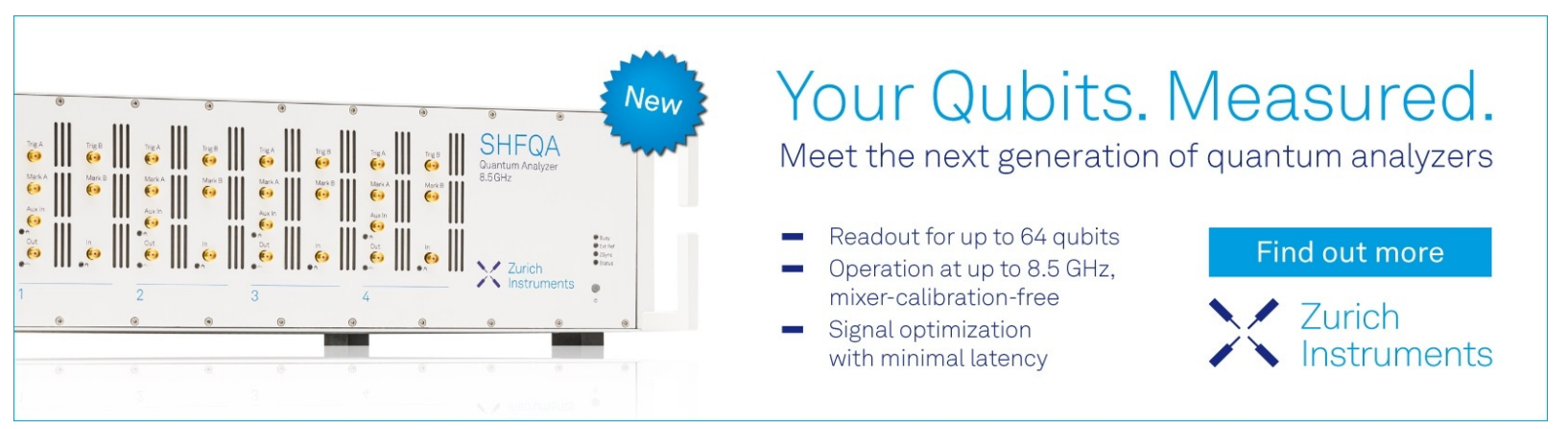




\title{
Super-chiral vibrational spectroscopy with metasurfaces for high-sensitive identification of alanine enantiomers
}

\author{
Cite as: Appl. Phys. Lett. 117, 101103 (2020); doi: 10.1063/5.0012331 \\ Submitted: 30 April 2020 - Accepted: 31 August 2020 • \\ Published Online: 9 September 2020

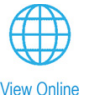 \\ Takumi lida, ${ }^{1}$ Atsushi Ishikawa, ${ }^{1,2,3}$ (D) Takuo Tanaka,, 2,3,4 (D) Atsuya Muranaka, ${ }^{5,6}$ (D) Masanobu Uchiyama, ${ }^{5,6}$ (iD \\ Yasuhiko Hayashi, ${ }^{1}$ (D) and Kenji Tsuruta, ${ }^{1, a)}$ (D)

\begin{abstract}
AFFILIATIONS
'Department of Electrical and Electronic Engineering, Okayama University, Okayama, Okayama 700-8530, Japan

${ }^{2}$ Metamaterials Laboratory, RIKEN Cluster for Pioneering Research, Wako, Saitama 351-0198, Japan

${ }^{3}$ Innovative Photon Manipulation Research Team, RIKEN Center for Advanced Photonics, Wako, Saitama 351-0198, Japan

${ }^{4}$ Institute of Post-LED Photonics, Tokushima University, Tokushima, Tokushima 770-8506, Japan

${ }^{5}$ Advanced Elements Chemistry Laboratory, RIKEN Cluster for Pioneering Research, Wako, Saitama 351-0198, Japan

${ }^{6}$ Craduate School of Pharmaceutical Sciences, The University of Tokyo, Bunkyo-ku, Tokyo 113-0033, Japan
\end{abstract}

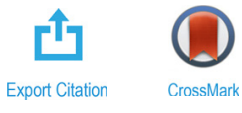

a) Author to whom correspondence should be addressed: tsuruta@okayama-u.ac.jp

\begin{abstract}
Chiral nature of an enantiomer can be characterized by circular dichroism (CD) spectroscopy, but such a technique usually suffers from weak signal even with a sophisticated optical instrument. Recent demonstrations of plasmonic metasurfaces showed that chiroptical interaction of molecules can be engineered, thereby greatly simplifying a measurement system with high sensing capability. Here, by exploiting super-chiral field in a metasurface, we experimentally demonstrate high-sensitive vibrational CD spectroscopy of alanine enantiomers, the smallest chiral amino acid. Under linearly polarized excitation, the metasurface consisting of an array of staggered Au nano-rods selectively produces the left- and right-handed super-chiral fields at $1600 \mathrm{~cm}^{-1}$, which spectrally overlaps with the functional group vibrations of alanine. In the Fourier-transform infrared spectrometer measurements, the mirror symmetric CD spectra of D-and L-alanine are clearly observed depending on the handedness of the metasurface, realizing the reliable identification of small chiral molecules. The corresponding numerical simulations reveal the underlying resonant chiroptical interaction of plasmonic modes of the metasurface and vibrational modes of alanine. Our approach demonstrates a high-sensitive vibrational CD spectroscopic technique, opening up a reliable chiral sensing platform for advanced infrared inspection technologies.
\end{abstract}

Published under license by AIP Publishing. https://doi.org/10.1063/5.0012331

Identification of amino acid enantiomers is the basis of asymmetric synthesis, playing an important role in the field of organic/ biological chemistry and pharmacy. ${ }^{1}$ Circular dichroism (CD), which is the differential absorption of left and right circularly polarized light, has been widely used to analyze the absolute configuration of chiral molecules. ${ }^{2-4}$ Depending on the different molecular excitations, enantiomers show the mirror symmetric CD spectra with an equal amplitude but opposite sign. Vibrational CD (VCD) spectroscopy in the infrared (IR) region measures the $\mathrm{CD}$ responses originating from vibrational excitations, whereas electronic $\mathrm{CD}$ (ECD) spectroscopy in the ultraviolet and visible regions measures those originating from electronic excitations. ${ }^{5,6}$ Compared to ECD spectroscopy, VCD spectroscopy allows one to analyze the detailed stereostructure of almost all chiral molecules without relying on chromophores. However, VCD spectroscopy usually suffers from weak signal, which is $\sim 10^{3}$ times smaller than common IR absorptions; therefore, the identification of small molecules with a high signal-to-noise $(S / N)$ ratio remains a major challenge.

Surface-enhanced IR absorption (SEIRA) by plasmonic nanostructures has been traditionally demonstrated for the high-sensitive analysis of molecular functional groups. ${ }^{7}$ Different from conventional metal island films, engineered plasmonic nanoantennas, i.e., metasurfaces, were also proposed for the resonant SEIRA to achieve atto/ zeptomole sensitivity with a large $\mathrm{S} / \mathrm{N}$ ratio. ${ }^{8-15}$ Inspired by such an 
approach, researchers have recently explored another sensing capability where $\mathrm{CD}$ response stems from the chiroptical interaction mediated by the local electromagnetic field. ${ }^{16-21}$ There, the chiroptical interaction of molecules is specified not only by the inherent material parameter, i.e., the dissymmetry factor, but also by the optical chirality, $\hat{C}=-\frac{c}{\left|E_{0}\right|^{2}} \operatorname{Im}\left(E^{*} \cdot \mathbf{B}\right),{ }^{21}$ where $c$ is the speed of light, $E_{0}$ is the incident electric field, and $\mathrm{E}^{*}$ and $\mathrm{B}$ are the complex conjugate electric and magnetic field vectors, respectively. The enhanced chiral electromagnetic field, the so-called super-chiral field, is then generated by the coupling of out-of-phase local electric and magnetic fields, leading to strong chiroptical interactions with molecules. ${ }^{22,23}$ With different types of plasmonic nanostructures, super-chiral spectroscopy has been experimentally demonstrated in the ultraviolet and visible regions, but few examples fully explored super-chiral vibrational spectroscopy, especially for the identification of small molecules. ${ }^{24-27}$

Here, we demonstrate high-sensitive VCD spectroscopy of alanine enantiomers by utilizing the resonant chiroptical coupling of the super-chiral modes of a metasurface and vibrational modes of alanine. Under linearly polarized excitation, the left- and right-handed super-chiral fields are selectively excited in the metasurface consisting of an array of staggered nano-rods. Different from conventional approaches with circularly polarized light, standard IR spectroscopy is then used to characterize the metasurface-molecular coupled system, greatly simplifying a measurement system with better sensitivity. Our approach demonstrates a prompt/easy-to-use chiral sensing technique of small molecules, holding great promise for a reliable sensing platform of advanced IR inspection technologies.

Figure 1(a) illustrates the schematic unit cell of the fabricated metasurface on a Si substrate consisting of an Au nano-rod pair with a longitudinal displacement, i.e., staggered nano-rods. The fabrication process started with the spin-coating of a 100-nm thick poly(methyl methacrylate) (PMMA) resist (MicroChem, 950-A2) onto a 325- $\mu \mathrm{m}$ thick double-side polished non-doped Si substrate. A two-dimensional periodic pattern of the metasurface was exposed by an electron beam (EB) lithography system (Elionix, ELS-S50) over an area of $500 \times 500$ $\mu \mathrm{m}^{2}$. The sample was then completed by $\mathrm{Cr} / \mathrm{Au}(3 / 15 \mathrm{~nm})$ deposition

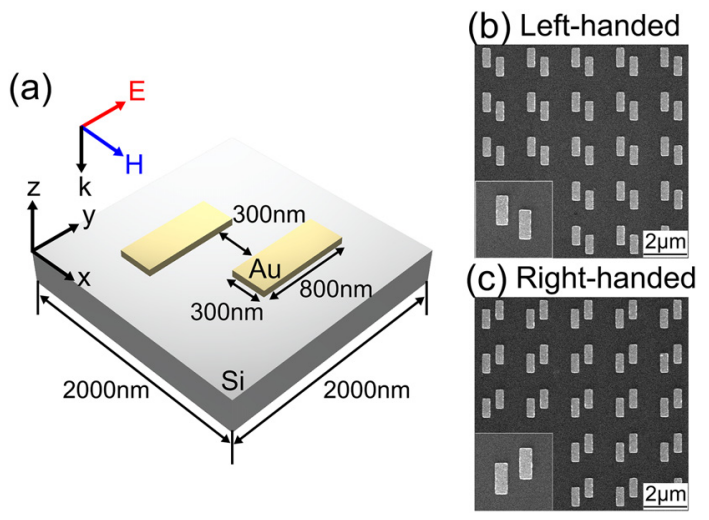

FIG. 1. Design and fabrication of the metasurfaces. (a) Schematic unit cell of a metasurface on a Si substrate consisting of an Au nano-rod pair with a longitudinal displacement. SEM images of the fabricated (b) left- and (c) right-handed metasurfaces. Depending on its handedness, the metasurface selectively produces the left/ right-handed super-chiral field at $1600 \mathrm{~cm}^{-1}$, which spectrally overlaps with the functional group vibrations of alanine. using a resistive heating evaporator and liftoff process. As shown in Figs. 1(b) and 1(c), the left- and right-handed structures were fabricated where the left or right nano-rod was shifted half along the y-axis. Depending on the handedness, the metasurface selectively produces the left/right-handed super-chiral fields at $1600 \mathrm{~cm}^{-1}$, which spectrally overlaps with the functional group vibrations of alanine. ${ }^{28,29}$

The optical activity of the metasurface was first characterized by using a Fourier-transform infrared spectrometer (FT-IR) equipped with a polarized infrared microscope (JASCO, FT/IR-6300FV and VIRT-3000). As shown in Fig. 2(a), two identical polarizers (JASCO, PL-82) with a high extinction ratio of $1 \times 10^{-2}$ over the measurement range were aligned before and behind the sample. To improve the S/N ratio of the spectra, a square aperture with an area of $500 \times 500 \mu \mathrm{m}^{2}$ was installed at the image plane of the microscope and a sample chamber was purged with a dry nitrogen gas. The measured spectrum was normalized by the corresponding result for the bare Si substrate to discuss only the optical response of the metasurface. Figure 2(b) shows the measured transmission spectrum of the left-handed metasurface at the analyzer angle of $\theta=0^{\circ}$. A distinct transmission dip due to a plasmon resonance of the staggered nano-rods was clearly observed at $1600 \mathrm{~cm}^{-1}$. The optical activity of the metasurface was then evaluated by measuring the differential transmission spectra, $\Delta T=T_{L}-T_{R}$, where $T_{L}$ and $T_{R}$ are the transmittance of the left- and right-handed metasurfaces, respectively. Figure 2(c) shows the measured $\Delta T$ at different angles of $\theta=-60^{\circ} \sim 60^{\circ}$ where the optical activity was increased at the resonance. These observations are well supported by the corresponding numerical results in Figs. 2(d) and 2(e), which were obtained by using the finite element method (FEM) software package, COMSOL Multiphysics, with $\varepsilon_{\mathrm{Si}}=11.56$ and the empirical value for

(a)

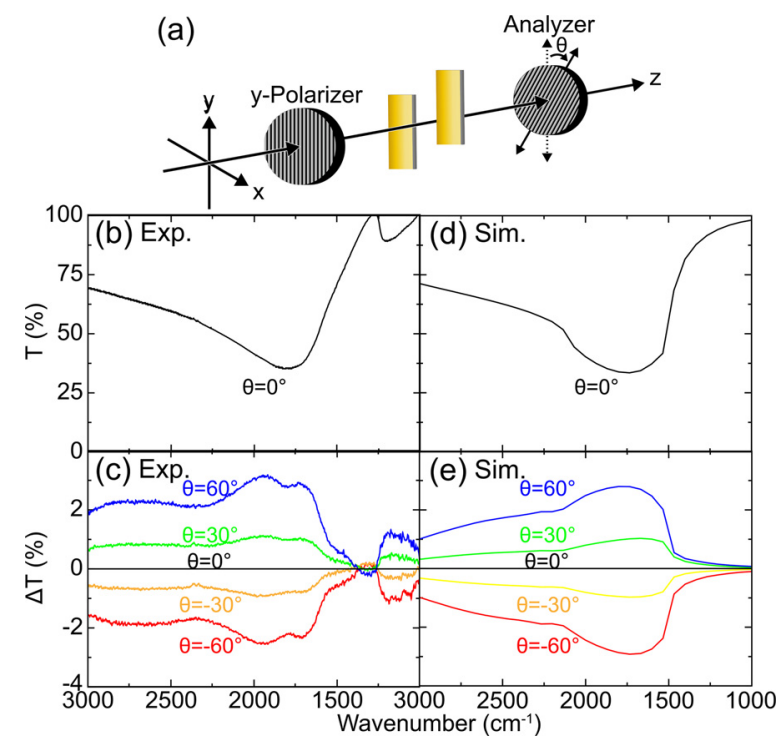

FIG. 2. IR characterization of the metasurfaces. (a) Experimental setup of the polarized FT-IR transmission measurement to evaluate the optical activity of the metasurface. (b) Experimentally measured transmission spectrum of the left-handed metasurface at the analyzer angle of $\theta=0^{\circ}$. (c) Experimentally measured differential transmission spectra between the left- and right-handed metasurfaces. (d) and (e) Corresponding numerical simulations, which well reproduced the experimental results qualitatively and quantitatively. 
$\varepsilon_{\mathrm{Au}} \cdot{ }^{30}$ Note that a minor plasmon resonance at $1200 \mathrm{~cm}^{-1}$ in Fig. 2(b) also induced a weak optical activity in Fig. 2(c), which was not reproduced in the numerical results [Figs. 2(d) and 2(e)]. The discrepancy can be explained by the fabrication/measurement error to induce the additional excitation of a plasmon mode. These results demonstrate that our metasurfaces exhibit strong optical activity depending on the handedness of the nano-rods. ${ }^{31,32}$

To better understand the underlying chiral property of the metasurface, another set of numerical simulations was carried out to evaluate the optical chirality, $\hat{C}$, in the vicinity of the nano-rods. Figure 3(a) shows the numerically simulated $\hat{C}$ in the left- (red) and right-handed (blue) metasurfaces measured at the gap of the nano-rods. The mirror symmetric spectral responses of $\hat{C}$ were clearly observed over the measurement range, showing a distinct enhancement peak at $1600 \mathrm{~cm}^{-1}$ in accordance with the plasmon resonance in Fig. 3(b). Figures 3(c) and 3 (d) show the corresponding $\hat{C}$ distributions in the left- and right-handed metasurfaces, where the sign of $\hat{C}$ was flipped depending on the handedness under the linearly polarized excitation. For the ypolarized incident wave, a dipole plasmon mode is excited in individual nano-rods with the maxima of electric (magnetic) field at the ends (center) of the nano-rods. ${ }^{17,23,33}$ By shifting one of the nano-rods half along the $y$-axis, the localized electric and magnetic fields overlap spatially and spectrally with a phase shift of $\pi / 2$ at the resonance, thereby producing an extra super-chiral field at the gap of the nano-rods. As a result, the overall optical chirality in the system has a finite value, which translates into the optical activity in the far-field measurement of Fig. 2(c). Only the molecules within the gap contribute to a molecular signal from the system, while the signals from the corner of the
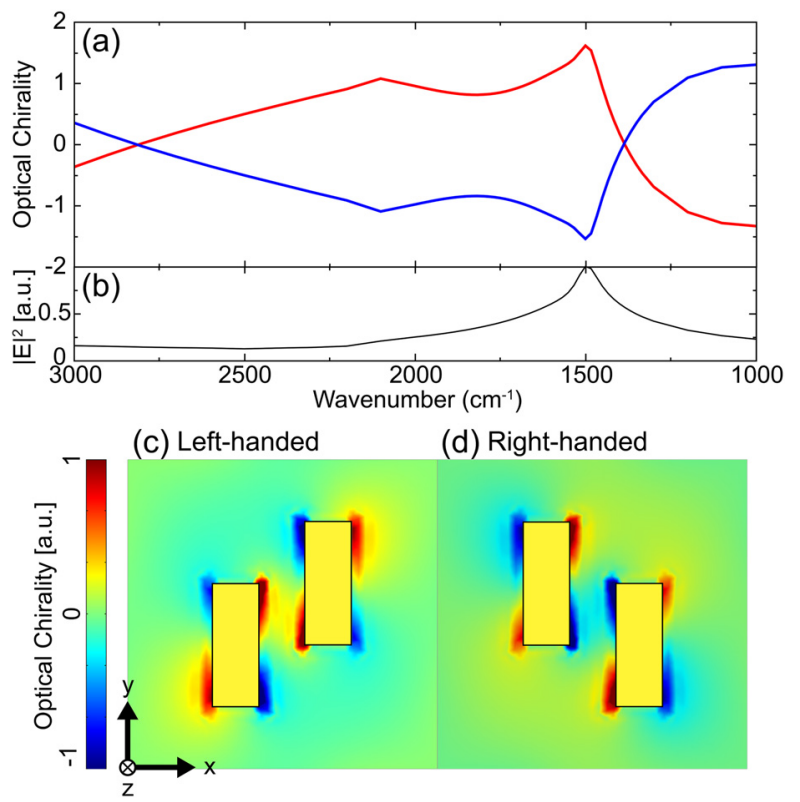

FIG. 3. Super-chiral field in the metasurfaces. (a) Numerically simulated optical chirality, $\hat{C}$, in the left- (red) and right-handed (blue) metasurfaces measured at the gap center of the nano-rods and (b) the corresponding $|E|^{2}$ spectral response. Corresponding $\hat{C}$ distributions in the (c) left- and (d) right-handed metasurfaces at $1600 \mathrm{~cm}^{-1}$, revealing that the sign of $\hat{C}$ was flipped between the left- and righthanded metasurfaces under the $y$-polarized excitation. nano-rods are eventually canceled out due to the point symmetric distribution of the optical chirality.

Since the left(right)-handed metasurface selectively interacts with a $\mathrm{D}(\mathrm{L})$-enantiomer, our approach offers a simple yet powerful IR sensing platform of chiral molecules. We first carried out a set of numerical simulations to demonstrate the underlying chiroptical interaction in the metasurface-enantiomer coupled system, as shown in Fig. 4. In the calculation, the optical response of chiral molecules was modeled as a Lorentz oscillator with the dielectric function: $\varepsilon_{r}=\varepsilon_{r 0}-\gamma$ $\left(\frac{1}{\hbar \omega-\hbar \omega_{0}-i \Gamma}\right)$ and the Pasteur parameter: $\kappa=\beta\left(\frac{1}{\hbar \omega-\hbar \omega_{0}-i \Gamma}\right)$, where $\varepsilon_{r 0}$ is the background relative permittivity, $\hbar$ is the Planck constant, $\omega_{0}$ is the resonance frequency, and $\Gamma$ is the damping constant. ${ }^{21}$ Such a modeling has been extensively investigated and widely applied in different systems. ${ }^{34-37}$ Here, the parameters, $\varepsilon_{r 0}=1.0, \gamma=2.0 \times 10^{-22}$, $\beta=7.5 \times 10^{-23}, \hbar \omega_{0}=1.98 \times 10^{-1} \mathrm{eV}$, and $\Gamma=1.56 \times 10^{-3} \mathrm{eV}$ were appropriately chosen such that the oscillator model emulated the resonant chiral response at $1600 \mathrm{~cm}^{-1}$, one of the vibrational modes of alanine. Figure 4(a) shows the spectral responses of $\kappa$ used in the simulations, and the sign was defined depending on the handedness of the enantiomer. Here, a relatively large $\operatorname{Re}[\kappa]=10^{-1} \sim 10^{-2}$ was used, but it should be a reasonable assumption for dense polycrystalline enantiomers under the on-resonant condition, compared to the offresonant one of $\operatorname{Re}[\kappa]=10^{-3} \sim 10^{-4}$. 38

An enantiomer on the left- and right-handed metasurfaces shows the differential absorption $\Delta A$ proportional to the optical chirality $\hat{C}$, which translates into differential transmittance in the far-field measurement. ${ }^{39}$ Figure 4(b) shows the numerically simulated spectra of D- (red) and L-enantiomers (blue) on the metasurfaces under the $y$-polarized excitation without the analyzer, demonstrating the mirror symmetric peak and dip, i.e., bisignate curves. Here, the spectra were obtained by calculating the differential transmission spectra, $\Delta T^{D(L)}=T_{L}^{D(L)}-T_{R}^{D(L)}$, where $T_{L}^{D(L)}$ and $T_{R}^{D(L)}$ are the transmittance of the left- and right-handed metasurfaces with $\mathrm{D}(\mathrm{L})$-enantiomer, respectively. Major responses at $1600 \mathrm{~cm}^{-1}$ arise from the $\mathrm{CD}$ responses of the enantiomers (the imaginary part of $\kappa$ ), while minor responses at $1500 / 2200 \mathrm{~cm}^{-1}$ arise from the coupling of the

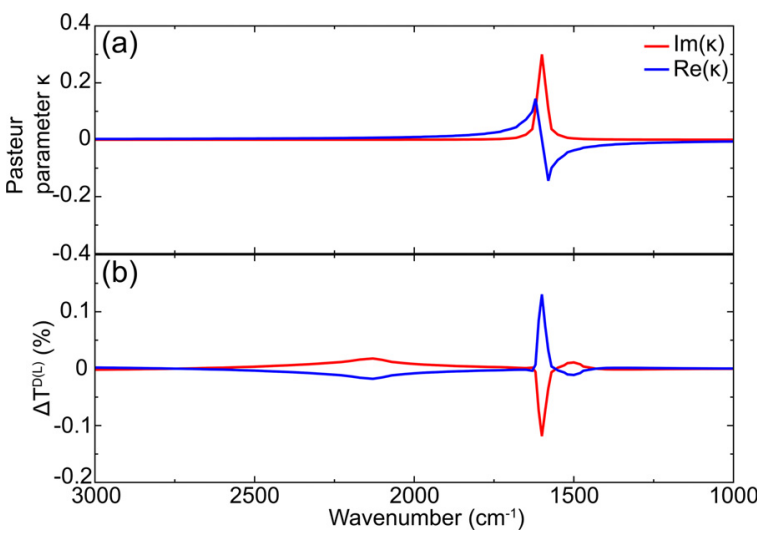

FIG. 4. Chiroptical interaction of a metasurface-enantiomer coupled system. (a) Spectral responses of the Pasteur parameter $\kappa$ of the enantiomer used in (b), exhibiting the resonant chiral response at $1600 \mathrm{~cm}^{-1}$. (b) Numerically simulated differential transmission spectra of D- (red) and L-enantiomer (blue) on the metasurfaces, which qualitatively reproduced the mirror symmetric peak and dip. 
metasurfaces and the optical rotation of the enantiomers (the real part of $\kappa$ ). These results reveal the underlying resonant chiroptical interaction of the metasurfaces and chiral molecules, leading to the enhanced sensing capability in the super-chiral VCD spectroscopy.

Based on the aforementioned chiroptical interaction and the resultant spectral responses of the system, we experimentally explored high-sensitive VCD spectroscopy of alanine enantiomers in the metasurface under linearly polarized excitation. Using a resistive heating evaporator, D- and L-alanine (Wako Pure Chemical Industries) were deposited onto the sample with a thickness of $50 \mathrm{~nm}$ to fully cover the surface structures. ${ }^{26}$ They spontaneously formed polycrystalline aggregates on the surface consisting of zwitterionic structures, as shown in the inset of Fig. 5(b), ${ }^{28,40}$ while linear dichroism arising from the anisotropy of the alanine aggregates was negligibly small in our work. Figure 5(a) shows the measured transmission spectrum of L-alanine on a bare Si substrate, demonstrating typical vibrational absorptions of the functional groups, $\mathrm{COO}, \mathrm{NH}_{3}, \mathrm{CH}_{3}$, and $\mathrm{CH}^{28,29}$ Note that the same spectrum was also observed for the D-alanine sample (not shown here), and enantiomer contamination was carefully avoided by cleaning the sample with the $\mathrm{UV} / \mathrm{O}_{3}$ ashing process after each measurement.

Figure 5(b) shows the measured $\Delta T^{D(L)}$ spectra of D- (red) and L-alanine (blue) based on the super-chiral vibrational spectroscopy in the metasurfaces under the y-polarized excitation without the analyzer. Note that the enantiomer correction was applied, where $\Delta T^{D(L)}$ were corrected to satisfy $\left(\Delta T^{D}+\Delta T^{L}\right) / 2=0$, producing the mirror symmetric spectral responses. Depending on the handedness of the metasurface, the super-chiral field selectively interacts with D- or L-alanine; therefore, $\Delta T^{D}$ and $\Delta T^{L}$ naturally show bisignate curves with large amplitudes at the resonance. The peak and dip frequencies were well matched with the corresponding functional group vibrations in Fig. 5(a). This observation was also supported by the VCD spectra of D- and L-alanine $\mathrm{D}_{2} \mathrm{O}$ solutions (Fig. $\mathrm{S} 1$ in the supplementary material), although their intensities were naturally different due to different vibrational modes between the liquid and solid states.

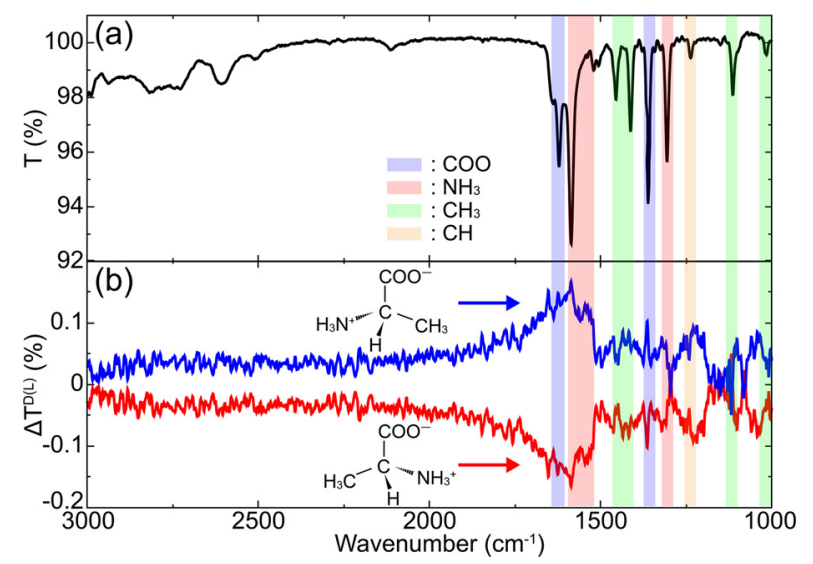

FIG. 5. Super-chiral vibrational spectroscopy in the metasurfaces. (a) Transmission spectrum of L-alanine on a bare Si substrate, exhibiting typical vibrational absorptions of the functional groups, $\mathrm{COO}, \mathrm{NH}_{3}, \mathrm{CH}_{3}$, and $\mathrm{CH}$. (b) Differential transmission spectra of D- (red) and L-alanine (blue) on the metasurfaces, showing the mirror symmetric spectral responses. The peak and dip frequencies were well matched with the corresponding functional group vibrations in (a).
Interestingly, $\Delta T^{D(L)}$ finely increased and decreased depending on the vibrational modes, and such a spectral feature may include the highorder chiral responses observed by the steep super-chiral field. The spectra obtained here are the result of the resonant chiroptical coupling of the metasurfaces and alanine enantiomers; thus, they are naturally different from the typical VCD spectra of the isolated crystalline alanine. ${ }^{29}$ Note that it has been shown that linear birefringence stems from the chiral property of the staggered Au nano-rods had no significant effect in the measurements. ${ }^{23}$ The reproducibility of our approach was also demonstrated in the additional measurements of the different samples (Fig. S2 in the supplementary material).

Conventional VCD spectroscopy usually suffers from weak signal, thus requiring a long integration time, e.g., a few hours for small molecules. However, our approach with the super-chiral field strongly enhances the chiroptical interaction of molecules, thereby dramatically reducing the measurement time. In the measurements, each spectrum with a reliable $\mathrm{S} / \mathrm{N}$ ratio could be obtained within a minute, much shorter than that of conventional techniques, even for small amounts of molecules. The enhanced sensitivity may offer a great advantage of studying the time-dependent CD responses of molecular conformational change. The measurement range of conventional techniques is usually limited by solvents for target molecules and optical components of an instrument. A simplified optical system in this work may also extend the measurement range inaccessible to conventional techniques, greatly enhancing the application of VCD spectroscopy.

In conclusion, we experimentally demonstrated super-chiral VCD spectroscopy of alanine enantiomers with a metasurface. The left- and right-handed super-chiral fields were selectively excited in the staggered nano-rod array by linearly polarized light. In the FT-IR measurements, mirror symmetric $\mathrm{CD}$ spectra of $\mathrm{D}$ - and L-alanine were clearly observed in the left- and right-handed metasurfaces, realizing the reliable identification of small chiral molecules. Our approach achieved a high-sensitive VCD spectroscopic technique, making an important step toward a reliable chiral sensing platform for advanced IR inspection technologies.

See the supplementary material for the VCD measurement of Dand L-alanine $\mathrm{D}_{2} \mathrm{O}$ solutions and the reproducibility of the measurements.

\section{AUTHORS' CONTRIBUTIONS}

A.I. initiated and managed the project. T.I. performed the sample fabrication and characterization, and the numerical simulation. All the authors discussed the results and contributed to the manuscript.

This work was supported, in part, by the JSPS KAKENHI Grant No. 17H06173 to M.U. The nanofabrication in this work was performed at the Division of Instrumental Analysis, Okayama University.

\section{DATA AVAILABILITY}

The data that support the findings of this study are available from the corresponding author upon reasonable request.

\section{REFERENCES}

${ }^{1}$ R. Noyori, Asymmetric Catalysis in Organic Synthesis (John Wiley \& Sons, New York, 1994). 
${ }^{2}$ Circular Dichroism and the Conformational Analysis of Biomolecules, edited by G. D. Fasman (Springer, New York, 1996).

${ }^{3}$ L. D. Barron, Molecular Light Scattering and Optical Activity (Cambridge University Press, 2004).

${ }^{4}$ I. F. Gallardo and L. J. Webb, Langmuir 28, 3510 (2012).

${ }^{5}$ T. B. Freedman, X. Cao, R. K. Dukor, and L. A. Nafie, Chirality 15, 743 (2003).

${ }^{6}$ P. L. Polavarapu and J. He, Anal. Chem. 76, 61 A (2004).

${ }^{7}$ M. Osawa and M. Ikeda, J. Phys. Chem. 95, 9914 (1991).

${ }^{8}$ F. Neubrech, A. Pucci, T. W. Cornelius, S. Karim, A. García-Etxarri, and J. Aizpurua, Phys. Rev. Lett. 101, 157403 (2008).

${ }^{9}$ C. Wu, A. B. Khanikaev, R. Adato, N. Arju, A. A. Yanik, H. Altug, and G. Shvets, Nat. Mater. 11, 69 (2012).

${ }^{10}$ D. Dregely, F. Neubrech, H. Duan, R. Vogelgesang, and H. Giessen, Nat. Commun. 4, 2237 (2013).

${ }^{11}$ K. B. Alici and I. F. Gallardo, Sci. Rep. 3, 2956 (2013).

${ }^{12}$ D. Rodrigo, O. Limaj, D. Janner, D. Etezadi, F. J. García de Abajo, V. Pruneri, and H. Altug, Science 349, 165 (2015).

${ }^{13}$ A. Ishikawa and T. Tanaka, Sci. Rep. 5, 12570 (2015).

${ }^{14}$ A. Ishikawa, S. Hara, T. Tanaka, Y. Hayashi, and K. Tsuruta, Sci. Rep. 7, 3205 (2017).

${ }^{15}$ A. Ishikawa, S. Hara, T. Tanaka, X. Zhang, and K. Tsuruta, Appl. Phys. Lett. 111, 243106 (2017).

${ }^{16}$ Y. Tang and A. E. Cohen, Phys. Rev. Lett. 104, 163901 (2010).

${ }^{17}$ E. Hendry, R. V. Mikhaylovskiy, L. D. Barron, M. Kadodwala, and T. J. Davis, Nano Lett. 12, 3640 (2012).

${ }^{18}$ M. Schäferling, X. Yin, and H. Giessen, Opt. Express 20, 26326 (2012).

${ }^{19}$ M. Schäferling, D. Dregely, M. Hentschel, and H. Giessen, Phys. Rev. X 2 , 031010 (2012).

${ }^{20}$ T. J. Davis and E. Hendry, Phys. Rev. B 87, 085405 (2013).

${ }^{21}$ M. L. Nesterov, X. Yin, M. Schäferling, H. Giessen, and T. Weiss, ACS Photonics 3, 578 (2016).

${ }^{22}$ Y. Tang and A. E. Cohen, Science 332, 333 (2011).
${ }^{23}$ N. Meinzer, E. Hendry, and W. L. Barnes, Phys. Rev. B 88, 041407 (2013).

${ }^{24}$ E. Hendry, T. Carpy, J. Johnston, M. Popland, R. V. Mikhaylovskiy, A. J. Lapthorn, S. M. Kelly, L. D. Barron, N. Gadegaard, and M. Kadodwala, Nat. Nanotechnol. 5, 783 (2010).

${ }^{25}$ R. Tullius, A. S. Karimullah, M. Rodier, B. Fitzpatrick, N. Gadegaard, L. D. Barron, V. M. Rotello, G. Cooke, A. Lapthorn, and M. Kadodwala, J. Am. Chem. Soc. 137, 8380 (2015).

${ }^{26}$ J. García-Guirado, M. Svedendahl, J. Puigdollers, and R. Quidant, Nano. Lett. 18, 6279 (2018).

${ }^{27}$ R. Knipper, V. Kopecký, U. Huebner, J. Popp, and T. G. Mayerhöfer, ACS Photonics 5, 3238 (2018).

${ }^{28}$ M. Tulio, S. Rosado, M. Leonor, R. S. Duarte, and R. Fausto, J. Mol. Struct. 410-411, 343 (1997).

${ }^{29} \mathrm{~S}$. Jähnigen, A. Scherrer, R. Vuilleumier, and D. Sebastiani, Angew. Chem., Int. Ed. 57, 13344 (2018).

${ }^{30}$ P. B. Johnson and R. W. Christy, Phys. Rev. B 6, 4370 (1972).

${ }^{31}$ M. Decker, M. Ruther, C. E. Kriegler, J. Zhou, C. M. Soukoulis, S. Linden, and M. Wegener, Opt. Lett. 34, 2501 (2009).

32J. K. Gansel, M. Thiel, M. S. Rill, M. Decker, K. Bade, V. Saile, G. von Freymann, S. Linden, and M. Wegener, Science 325, 1513 (2009).

${ }^{33}$ L. E. Barr, S. A. R. Horsley, I. R. Hooper, J. K. Eager, C. P. Gallagher, S. M. Hornett, A. P. Hibbins, and E. Hendry, Phys. Rev. B 97, 155418 (2018).

${ }^{34}$ A. O. Govorov, J. Phys. Chem. C 115, 7914 (2011).

${ }^{35}$ N. A. Abdulrahman, Z. Fan, T. Tonooka, S. M. Kelly, N. Gadegaard, E. Hendry, A. O. Govorov, and M. Kadodwala, Nano Lett. 12, 977 (2012).

${ }^{36} \mathrm{~W}$. Zhang, T. Wu, R. Wang, and X. Zhang, J. Phys. Chem. C 121, 666 (2017).

${ }^{37} \mathrm{~K}$. Yao and Y. Zheng, J. Phys. Chem. C 123, 11814 (2019).

${ }^{38}$ J. García-Guirado, M. Svedendahl, J. Puigdollers, and R. Quidant, Nano. Lett. 20, 585 (2020).

${ }^{39}$ M. Schäferling, N. Engheta, H. Giessen, and T. Weiss, ACS Photonics 3, 1076 (2016).

${ }^{40}$ H. J. Simpson, Jr. and R. E. Marsh, Acta Crystallogr. 20, 550 (1966). 